BNL 18057

Co.vf-730464--1

NEUTRON SCATTERING STUDIES OF SOFT MODE DYNAMICS

J. D. Axe, S. M. Shapiro, and G. Shirane

Brookhaven National Laboratory

Upton, New York 11973 U. S. A.

T. Riste

Research Establishment, Kjeller, Norway

At the NATO Advanced Study Institute held at nearby Ge1lo in Aprii 1971, Riste, Samuelsen, and Otnes ${ }^{1}$ reported on the critical behavior of $\mathrm{SrTHO}_{3}$ near the $105^{\circ} \mathrm{K}$ structural phase transformation as studied by neutron scattering. They observed that In addition to the expected condensing soft mode phonon side bands that there was in addition a very narrow central component. This paper is in the nature of a progress report on the neutron scattering work of the last two years on the "central mode problem."

\title{
B-TUNGSTEN SUPERCONDUCTORS
}

The first thing we learned was that the central mode was not an isolated phenomenon occurring only in $\mathrm{SrTiO}_{3}$. At about the same time, Shirane and I were studying the structural transformation in $\mathrm{Nb}_{3} \mathrm{Sn} .^{2}$ This structural transformation, which occurs in many binary or pseudoblnary compounds with the same $B$-tungsten structure, is characterized by a drastic softening of the acoustic shear mode with propagation vector $\vec{q}||[110]$ and displacement vector $\vec{e}||[1 \overline{1} 0] .3^{3}$ At the transition temperature $T_{M}=46^{\circ} \mathrm{K}$, the crystal structure changes from cubic to a slightly distorted tetragonal structure. ${ }^{4}$ Fig. 1 summarizes the frequency shifts seen

*Work performed under the auspices of the U. S. Atomic Energy Commission and NATO Research Grant. 


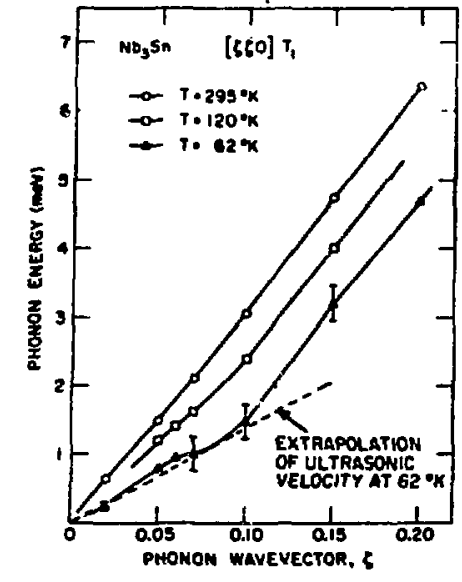

Neg.非 4-675-73

Figure 1. Temperature dependent transverse acoustic phonon dispersion at small wave vectors propagating in the "soft" [110] direction In $\mathrm{Nb}_{3} \mathrm{Sn}$.

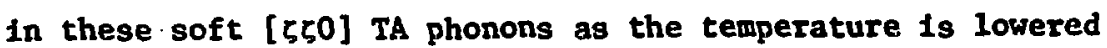
toward $T_{M}$. It is probable that the change in slope which occurs at $q \sim 0.1$ is related to the change in screening which occurs as the phonon wave vector passee through parallel edges of the Fermi surface. Most interesting for the purposes of the present discussion are the changes in the power spectrum of the soft phonons in the range of wave vector shown in Fig. 1 as the temperature is lowered further. Typical of these observations are the data in Fig. 2 showing that as the temperature is lowered there is a $8 \mathrm{rad}-$ ual evolution of a central component in the scattering spectrum in addition to the familiar "phonon-11ke" sidebands. Although the sideband structure continues to move to lower frequencies as the temperature is lowered, far more dramatic (note the logarithmic scale) is the growth of intensity of the central component which completely dominates the fluctuation spectrum near $\mathrm{T}_{\mathrm{M}}$. The apparent width of the central component can be essentially accounted for by the resolution of the instrument alone. Thus the intrinsic width which adds in quadrature to the instrumental width is small, $1 / 3$ or less of the observed width. FIg. 3 demonstrates that this central peak intensity maximizes at or very near $T_{M}$ and thus represents the major contribution to the critical scattering associated with the structural transformation. An additional qualitative observation to that although this additional central component can be observed at least out to $q=0.1$, 1ts 


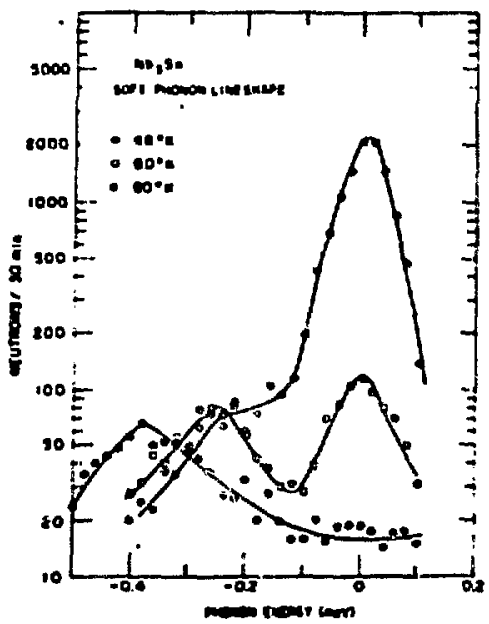

Neg. \# 4-676-73

FIgure 2. Observed spectral profiles of $[550] T_{i}$ phonon mode in $\mathrm{Mb}_{3} \mathrm{Sn}$ with $\zeta=0.02 \mathrm{a}^{\star}$ at several temperatures above $T_{M}$. Only the phonon annihllation portion of the spectrum is shown. The data were taken with an incoming neutron energy of 5 meV and the energy resolution is $\sim 0.1$ meV.

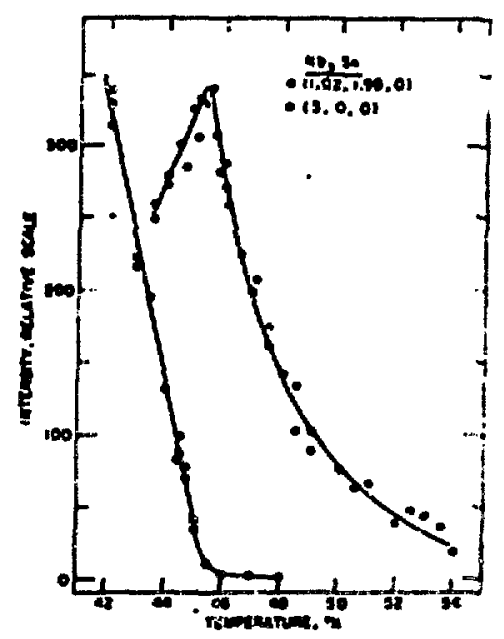

Neg.* 8-126-71

P1gure 3. The closed circles show the onset of the structural phase transformation as monitored by the "forbidden" (300) Bragg reflection. The open circles represent the tewperature dependence of the central component in the neutron crielcal scattering spectrum of $\mathrm{Nb}_{3} \mathrm{Sn}$. 
presence is restricted to propagation directions nearly along the soft [110] direction: Finally, by obserying, this feature for several values of momentum transfer $\vec{Q}-\vec{G}+\vec{q}$ which differed by only a reciprocal lattice vector $\vec{G}$, we found the Intensities of the central component and the phonon sidebands to be in a constant ratio. This seems to establish that both the central peak and the stdebands are describing the motion of one and the same mode, but that the mode dynamics are characterized by both the normal phonon-like osclllatory response and some slower response as well. (I w1ll discuss later the still-to-be-eliminated possibility that the central component is a purely static phenomenon.)

In order to discuss our observations in a quantitative way, 1t was necessary to Invent some $k$ ind of a theoretical construction, however tentative, and this we did in the following way. Quite generally. the frequency dependent part of the one-phonon ecattering criss section may be written as

$$
s(\omega)=\left(\frac{k_{B} T}{f_{1} \omega}\right) \operatorname{Im}\left(\omega_{0}^{2}-\omega^{2}-1 \omega r(\omega)\right\}^{-1}
$$

where $\omega_{0}$ is a temperature-dependent quasthermonic frequency and we assume $k_{B} T \gg$ tit for the frequency range of interest. Softmode IIne shapes have prevlously been discussed in the "viscous daming" approximation in which $r$ is taken to be a frequency1ndependent constant. The condition for dynamical instability, $\omega_{0} \rightarrow 0,1 s$ connected wich the divergence of the integrated scattering intensity I(total) - $\int S(\omega) d \omega \propto \omega_{0}^{-2}$. This form leads to efther a two- or one-peaked function, depending upon the ratio r/wo and is not capable of explaining even qualitatively the profilies shown in Fig. 2.

In general, however, I Itself has a frequency dependence which reflects the changing denalty of excitations with which the one-phonon state can Interact. Suffictently large changes in $r(\omega)$ In the Important frequency region near $\omega_{0}$ can produce more conplicated spectral profiles. We postulated the following simple form for the phonon self-energy function

$$
r(\omega)=r_{0}+\delta^{2} /(\gamma-1 \omega) \text {. }
$$

Schwabl later Independently proposed a ofullar Anate for $\Gamma(w)$ in discussing the central mode in $\mathrm{SrTHO}_{3} .5$ We Imigined the firat ters in Eq. (1) to represent the normal damping due to phononphonon scattering and the second term as due t:o coupling with another (as yet unspecified) fluctuation with. a Debye relaxation epectrum. Eq. (1) has been criticized on the grounds that it violates certain monent sum rules. This is related to the failure of $\Gamma(\omega) \rightarrow 0$ as $\omega \rightarrow \infty$. $r_{0}$ should be thought of as the low 
frequency approximation to a function $\Gamma_{0}(\omega)$ which varies slowly over the region $\omega \approx \dot{\omega}_{0} \ll \omega_{D}$, where $\omega_{D}$ is the Debye frequency of the solid. Thus in systems studied thus far it is mathematically correct but physically improper to omit $r_{0}$ in Eq. (1) on the basis that the remaining term satisfies higher monent sum rules.

Eq. (1) seems now to be established as the canonical line shape In the central mode problem. One would do well to remember however that it has a very slender foundation in microscopic theory. It has survived rather well whatever experimental tests we have subjected it to, but these have not been stringent enough to allow us to say that a correct theory must yleld this exact anslytical form.

Inserting Eq. (2) Into Eq. (1) gives

$$
s(\omega)-\frac{\left(\frac{k_{B} T}{h}\right)\left[r_{0}+\frac{\delta^{2} \gamma}{\omega^{2}+\gamma^{2}}\right]}{\left[\left(\omega_{\infty}^{2}-\frac{\delta^{2} \gamma^{2}}{\omega^{2}+\gamma^{2}}-\omega^{2}\right)^{2}+\omega^{2}\left(r_{0}+\frac{\delta^{2} \gamma^{2}}{\omega^{2}+\gamma^{2}}\right)^{2}\right]}
$$

where $\omega^{2}=\omega^{2}+\cdot \delta^{2}$. This formula has the general qualitative features of Fig. 2. In the limit $\omega_{s}^{2} \gg \delta^{\text {? }}$. 1t shows three distinct peaks with side bands at $\pm \omega_{\infty}$. In thi other 11att $\omega_{0} \rightarrow 0$ (1.e. $\left.\omega_{\infty} \rightarrow|\delta|\right)$, Eq. (3) shows a profile with shoulders similar to that observed in $\mathrm{Fig}_{\mathrm{g}} 2^{2}$ at $46^{\circ} \mathrm{K}$.

For a more quantitative comparison with experiment, we can conveniently divide the aross section into $S(w)_{\text {total }}=s(w)_{\text {central }}$ $+S(\omega)$ aldeband with.

$$
S(\omega)_{\text {centra! }}=\left(\frac{k_{B} T}{\pi}\right)\left(\frac{\delta^{2}}{\omega_{0}^{2} \omega_{0}^{2}}\right)\left(\frac{\gamma^{\prime}}{\omega^{2}+\gamma^{12}}\right)
$$

where $r^{\prime}=\left(\omega^{2} / \omega_{\infty}^{2}\right) Y$. This formula is valld for the range of parameters of ${ }^{0}$ taterest, $\omega_{\infty} \gg \gamma, \gamma$ and $\eta_{0} \ll<\left(\delta^{2} / \gamma\right)$. As with the elmpler damping, $I_{\text {tat }} * \omega_{0}^{-2}$ and dynamical Instability occurs as $\omega_{0} \rightarrow 0$. The fractional integrated central peak intensity is exply

$$
\frac{I(\text { central })}{I(\text { total })}=\frac{\delta^{2}}{\omega_{0}^{2}} \text {. }
$$

From data of the type shown in Fig. 1, it 1s straightforward to obtain both the ratio I (central)/I(total) and the value of $w$ (essebtially the peak of the phonon sideband), so that Eq. (5) 
can be used to deduce a value of $|\delta|$. Investigation for various wave vectors establishes that $|\delta|$ varies approximately linearly with $q,|\delta|=\lambda \mathrm{q}$. The principal prediction of this phenomenological theory is that the central mode intensity grows not in proportion to the increasing intensity of the soft phonon sidebands $\left(\sim T / \omega_{\infty}^{2}\right)$ but rather at the much faster rate $\left(\sim T \delta^{2} / \omega_{\infty}^{2} \omega_{0}^{2}\right)$. The observed temperature dependence is consistent with this relation If $\delta$ is taken as temperature independent.

There is one additional inftially puzzling feature of our data which is resolved by our present understanding of the significance of the central mode. At room temperature the value of the $(550) \mathrm{T}_{1}$ phonon velocity derived from neutron messurements is essentially equal to that obtalned by ultrasonic techniques? However, as shown in Fig. 4 there is an increasing gystematic diocrepancy between the two types of meawirements. He may auppose that the ulirasonic frequency ( $40 \mathrm{kHz}$ in this ingtance) is negligibly small cempared with the inverse relaxation time $r$, in which case one measures a low frequency sound velocity $v_{0}$. On the other hand, phonon frequenctes from which the neutror. deterwined velocity is derived is much greater than $\gamma$, in which case the observed phonon velocity can be shown from Eq. (3) to be

$$
v_{0}^{2}=\left(\omega_{0}^{2} / q^{2}\right)=v_{0}^{2}+\lambda^{2}
$$

Plotted In Fig. 4 are the ultrasonic relocities $v$ and the values of $v_{0}$ calculated from Eq. (6), and using the val $48=6.42 \times 10^{4} \mathrm{~cm} /$ sec obtalned from the above analysis of the cencral peak amplitude. Vo agrers very well wich the velocities obtained from the direct analyols of the sideband frequencies obtained from the

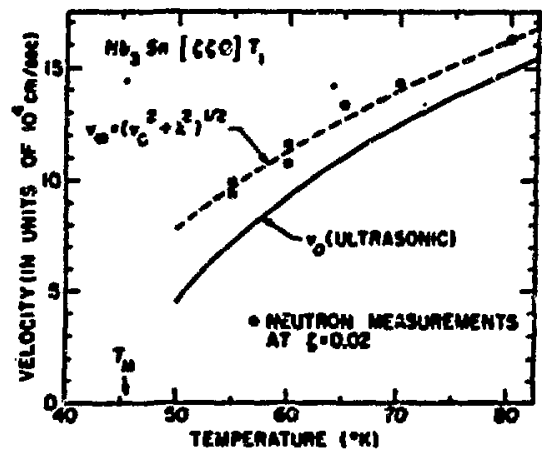

Neg. \#6-182-72

P1gure 4. The $(550) \mathrm{T}_{1}$ phonon velocity in $\mathrm{Mb}_{3} \mathrm{Sn}$ deternined by neutron acattering differs from uitrasonlc velocteles by an wount which is predictable from the anplitucie of the central component. 
neutron experiments.

$\mathrm{SrTIO}_{3}$

As is now well known, the gtructural phase transformation in $\mathrm{SrTHO}_{3}$ at $2105^{\circ} \mathrm{K}$ results fron the instablitty of a zone boundary phonon. ${ }^{7-9}$ In the cuble phase the displacements of the soft mode transform according to the triply degenerate $R_{25}$ representation of the group of $R$ and correspond to rotation of the oxygen tetrahedra about the $\langle 100\rangle$ axes. At $T_{c}$ there $1 \mathrm{~s}$ a doubling of the unit cell and the zone boundary of the cubic phase now becomes the zone center of the tet gonal phase. The triply degenerate zone-boundary $R_{25}$ mode of the cublc phase splits into two zonecenter modes: a doublet with rotation axes perpendicular to the fourfold axis and a singlet with rotation axis parallel to the fourfold axis. Subsequent to the dlocovery of the "central mode" in $\mathrm{SrTHO}_{3}$ by Riste et al kirther experiments were carried out in collaboration with Riste at the HFBR at Brookhaves. ${ }^{10}$

In the study of $\mathrm{NB}_{3} \mathrm{Sn}$ the small sample s1ze $\left(0.05 \mathrm{~cm}^{3}\right) \mathrm{se-}$ verely limited the scope of the study, but fortunately the extension of the central composent in reclprocal space was much larger than the corresponding q-width of the instrumental resolution. While the observed line shapes were st1ll subject to considerable correction for finite energy resolution, to within a reasonable firgt approximation the reiative integrated intensities of the central and sideband components were given directly from the experimental data. In the case of $\mathrm{SrTiO}_{3}$ (as well aq $\mathrm{KMnF}_{3}$ and $\mathrm{LaNO}_{3}$, to be discwsed later) the cross section was observed to vary rapidly over a range of q comparable to the Instrumental width. This necessitated resolution corrections, the Importance of which can be visualized with the ald of F1g. 5, which scheatically shows a cross section corresponding to a phonon disperalon surface, some additional cross section around $\omega=0$, and the resolution ellipse draw to scale for a typlcal high resolution experiment. A constant $Q$ scan woves the resoluition function parallel to the energy axis through the scattering cross section. For a particular setting $\left(Q_{s}, \omega_{s}\right)$ of the specrrometer the observed neutron intensity is

$$
I\left(Q_{s}, \omega_{a}\right)=f d \phi_{d \omega R}\left(\bar{Q}-\bar{Q}_{\mathbf{s}}, \omega-\omega_{8}\right) \sigma\left(Q_{0} \omega\right)
$$

and

$$
\sigma(\vec{Q}, \omega)=\frac{k_{q}}{k_{I}}\left|F_{1 n}(\vec{Q})\right|^{2} s_{q}(\omega)
$$

where $k_{F}$ and $k_{f}$ are the final and initial neutron wowente and 
F1gure 5. Schematic representation of a cross section of the soft phonon dispersion near the R-point in $\mathrm{SrTHO}_{3}$, with additional scattering near $\omega m 0$. The rasolution ellipse 1a drawn to scale for 5-meV incident niatron energy. $20^{\prime}$ horizontal collimetion.

$F_{1 n}(\vec{Q})$ Is the Ingelastic structure factor for the mode with a reduced momentum $\vec{q}=\vec{Q}-\vec{G}$.

The intensity of the phonon-like sidehands changes with $\vec{Q}$ much less rapldiy than does that of the central component, the two components are therefore welghted differently by the spectrometer. The proper resolution correction can be applied only if we knuw the $Q$ dependence of $O(\vec{Q}, w)$. If the dynamical matrix is expanded about $q_{B}=(1 / 2,1 / 2,1 / 2)$, the bahavior of the "bare" phonon, podes neat $\vec{q}_{R}$ are determined by a tzuncated dynamical extrix 11

$$
G_{T}(\vec{q})=\omega_{-}^{2}\left(\vec{q}_{R}\right) \underline{I}+\left(\vec{q}-\vec{q}_{R}\right) \cdot \lambda \cdot\left(\vec{q}-\vec{q}_{R}\right)
$$

where 1 is a $3 \times 3$ unit matrix and $\lambda$ contains the 3 independent constants which can be adjusted to agree with the measured frequencles $\omega_{s}^{2}(\vec{q})$. The Inelastic structure factors $F_{\text {in }}(\vec{q})$ are easily svaluated from the elgenvalues of Eq. (9). Although we can afely neglect the q-dependence of $\Gamma_{0}$, our phenomenological derlvation of $S_{q}(w)$ gives us no guide for predicting $\delta(\vec{q})$ and $r(\vec{q})$. Our analysis wili be independent of any detalled assumptions about $r(\vec{q})$, but in getting $\delta(\vec{q})$ - constant, we are in effect averaging whatever $\vec{q}$-dependence there is in this quantity over the sampled region of womentum transfer.

Fig. 6 is an example of the observed line shape at about ten degrees sbove $T_{c}$. Very high resolution was obtalned by using low energy (4.9 meV) Incident neutrons and higher enexgy contanination was reduced to a negligible level by a Be filter. The 
Neg. $4-18 \mathrm{i}-72$

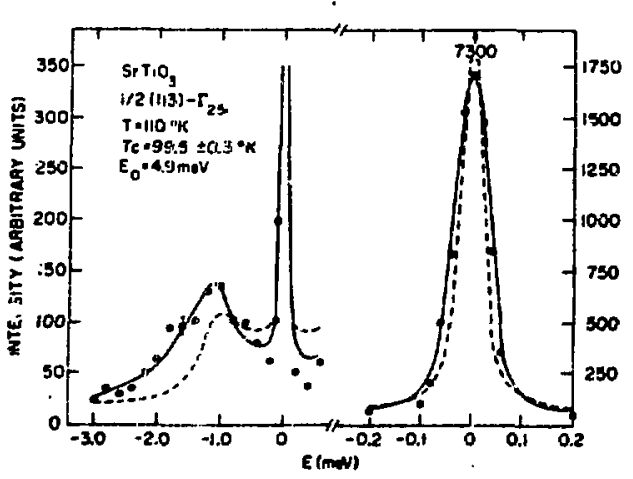

Figure 6. Scuttered-neutron spectrum of $\mathrm{SrTiO}_{3}$ at ? . $110^{\circ} \mathrm{K}$. The circles are the observed data, the full line represents the fit of the data with $\mathrm{Eq}$. (3) folded with the instrumental resolution; $\Gamma_{0}=0.88 \pm 0.1 \mathrm{mev}, \delta^{2}=0.63 \pm$ $0.1 \mathrm{meV}^{2}, \omega^{2}=1.27 \pm 0.1 \mathrm{meV}^{2}, \lambda_{1}=500 \mathrm{meV}^{2} \mathrm{~A}^{2}, \lambda_{2}=$ $1000 \mathrm{meV}^{2} \mathrm{~A}^{2}$. The dashed curve is a plot of $\mathrm{s}(\omega)$ with the above parameters.

Incoherent scattering (a small correction at this temperature) was measured separately and has been subtracted. The Importance of the resolution corrections, saen by comparing the solid and totted cusves is, as expected, most pronounced on the central peak.

Since the observed linewidth of the central peak was always resolution 1imited we chose a nominal value of $Y^{\prime}$ such that it was always smaller than the energy resolution of the spectrometer. After a good fit was obtained $\gamma^{\prime}$ was allowed to vary. The fit was Insensitive to $\gamma$ " provided it was smaller than the instrumental resolution and larger than the mesh size required for the numerical folding. As can be seen mogt easily by the approzimate $\mathrm{Eq}$. (4), $\delta^{2}$ can stil 1 be determined accurately under these conditions from the integrated central peak intensity.

F1g. 7 shows the temperature dependence of $\delta^{2}, \omega_{\infty}^{2}\left(\vec{q}_{R}\right)$ and $\omega_{0}^{2}\left(q_{R}\right)$ deduced by fitting many experimental results to $\mathrm{Eq}$. (3). Several internal checks, such as the consistency of parameters obtained with different spectrometer resolution as well as the overall goodness of fit, convince us that the form of the parimeterized cross section we have chosen 18 not grossly inappropriate. There are at least two features here worthy of comment. $\delta^{2}$ 1s apparently constant at $\sim 0.9 \mathrm{meV}^{2}$ for $T-T_{c}>25^{\circ} \mathrm{K}$, decreasing to about $0.3( \pm 0.1) \mathrm{meV}^{2}$ at $T_{c}$. It is possible, however, tiat all or part of this apparent I-dependence results from our rather ad hoc assumption that $\delta^{2}$ is q-independent. 


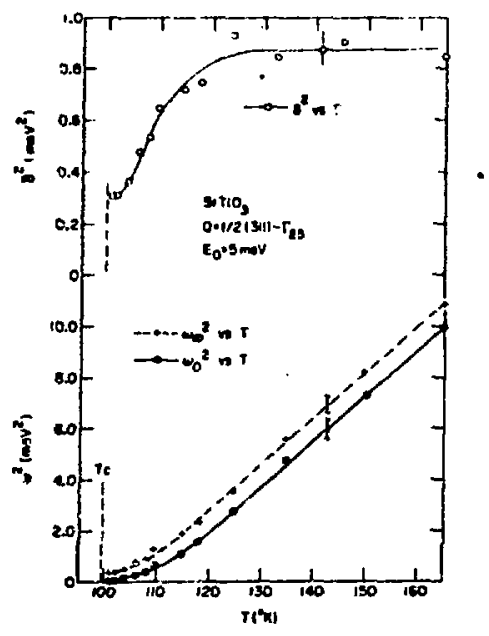

Figure 7. $6^{2}, \omega_{\infty}^{2}, \omega_{0}^{2}$ vs. $\mathrm{T}$ for $\mathrm{SrTHO}_{3}$.

In agreement. with previous studies $1,12 \omega^{2}$ is essentially IInear with temperature in the range $10^{\circ} \mathrm{K}<T^{\circ}-T<180^{\circ} \mathrm{K}$, but a deviation from this simple mean field behavior is clearly evident at lower temperatures for both $\omega_{\infty}^{2}$ and $\omega_{0}^{2}$. Since $\omega_{0}^{2}$ is essent1ally the inverse static susceptibility an attempt was made to deduce a critical exponent, $\gamma$, using the relation $\omega^{2}\left(T \rightarrow T_{c}\right) \sim\left(T-T_{c}\right)^{\gamma}$ but the results were not satisfactory, perhaps because of the ilmited temperature range of our observations. However, our measurements strongly suggest that if such a limiting form is applicable, $1.5<\gamma<2.5$. It was not possible to make meaningful measurements nearer $T$ because of our inability to determine $T$ to better than $\sim \pm 0.5^{\circ}$. This determination of $T$ was made by ofserving the intensity change of the cubic (222) reflection caused by extinction relief in the strained setragonal crystal.

\section{$\mathrm{RMnF}_{3}$}

$\mathrm{KMnI}_{3}$ exhibits the same cublc-tetragonal transformation that exists in $\mathrm{SrTHO}_{3}$ with $\mathrm{T}_{0}=186^{\circ} \mathrm{K} .13$ Gesi et al ${ }^{14}$ studied the temperature dependence of the soft mode line shape with moderately high resolution and were able to describe their observations with a simple damped harmonic osctllator response, but found an unexpected temperature dependence of the damping parameter $r_{0}$. This led us to a relnvestigation of $\mathrm{KMnF}_{3}$ with higher resolution and the observation of a narrow central component in addition to a broader overdamped phonon peak.10 


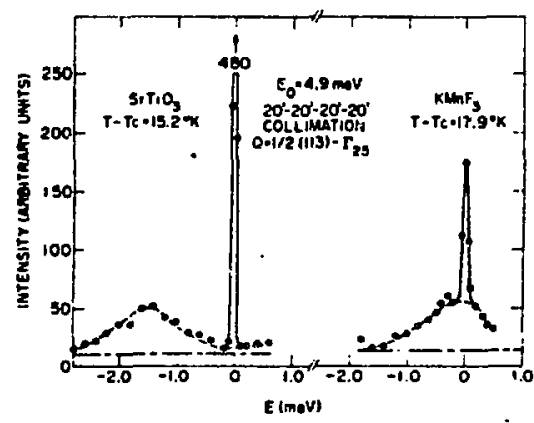

Figure 8. Scattered-neutron spectra of $\mathrm{SrTHO}_{3}$ and $\mathrm{KMnF}_{3}$ at $T-T_{c}=15.2$ and $17.9^{\circ} \mathrm{K}$, respectively. The dotted line corresponds to the phonon peak and the solid line to the central component. The dashed line corresponds to level of room background. All incoherent scattering has been subtracted.

Fig, 8 compares the soft phonon line shapes of $\mathrm{SrTHO}_{3}$ and $\mathrm{KMnF}_{3}$ under Identical high resolution and at nearly identical vaIues of $T-T_{c}$. It shows unambiguously the existence of the central peak in $\mathrm{KMnF}_{3}$ as well as in $\mathrm{SrTHO}_{3}$. In the latter, the -phonon peak (dotted line) is underdamped, whereas in $\mathrm{KMnF}_{3}$ the phonon is well overdamped at this value of $T-T_{c}$ and appears as a broad line centered around $\omega=0$. Sitting on top of this broad ine is the narrow central peak. In addition, the linewidth of the central peak in both systems is that of the instrumental resolution. This sets an upper limit on the value of the central component: $\gamma<0.02 \mathrm{meV}\left(=0.16 \mathrm{~cm}^{-1}=4.8 \times 10^{9} \mathrm{~Hz}\right)$.

Although there is no difficulty in fitting the line shape observed in $\mathrm{KMnF}_{3}$ to $\mathrm{Eq}$. (3), the results are considerably less rellable because severe correlations develop between the fitting parameters, particularly $\omega_{\infty}^{2}$ and $\delta^{2}$. If, however, we use the values of $\omega_{\infty}^{2}(T)$ given by Gesi et al (which should te reasonably rellable for $T$ not too close to $T_{c}$ ) values of $\delta^{2} \sim 0.3 \pm 0.1 \mathrm{mev}^{2}$ can be estimated.

If, In accordance with our assumption that $\delta(\vec{q})$ can be regarded as $\Varangle-$-independent, the central mode intensity depends upon वे only through $\omega_{\infty}(\bar{q})$, and from Eq. (4), it is given approximately by $\left[\omega_{\infty}^{2}(\bar{q}) \omega_{0}^{2}(\vec{q})\right]^{-1}$. $\omega_{\infty}^{2}(ఫ)$ is much more anisotropic in $\mathrm{KMnF} \mathrm{F}_{3}$, the soft branch being especially low along the Brillouin zone boundary from $R(1 / 21 / 21 / 2)$ to $M(1 / 21 / 20)$. We should therefore expect the central mode intensity to be more diffuse in $\mathrm{KMnF}_{3}$, extending especially in the direction $R \rightarrow M$. This indeed appears to be the case. 
$\mathrm{LaAlO}_{3}$

This perovskite material also has $(1 / 21 / 21 / 2)$ wave vector phonon instabillty ${ }^{14}$, but the rotation of the anton octahedra is about (111) axes rather than about $(100)$ as is the case for $\mathrm{SrTiO}_{3}$ and $\mathrm{KMnF}_{3}$. A recent reexamination of the soft mode line shape by $\mathrm{Kjems}$ et al ${ }^{15}$ establishes the existence of a central peak in this material as well. The damping of the phonon-like siflebands, although greater than for $\mathrm{SrTiO}_{3}$, was not found to be as large as in $\mathrm{MMnF}_{3}$, and made posstble a more extensive quantitative discussion. The collection and treatment of the experimental data was very similar to that for $\mathrm{SrTiO}_{3}$. The energy width was resolution limited, with an upper limit of $\gamma^{\prime} \leq 0.03 \mathrm{mev}$. Eq. (3) adequately represented the observed scattering and for $\mathrm{T}-\mathrm{T} \geq 15^{\circ} \mathrm{C}, \delta^{2}=0.38 \pm 0.1 \mathrm{meV}^{2}$ and was nearly temperature independent. As was the case in $\mathrm{SrTHO}_{3}, \omega^{2}$ and $\omega_{\infty}^{2}$ exhibited linear temperature dependence well away from $T_{\text {but }}$ the extrapolated values go to zero well above the observed transformation temperature, and the observed frequencles deviate considerably from the mean field behavior near $T \cdot$. The behavior of both $\omega_{\infty}^{2}(T)$ and $\delta^{2}(T)$ are shown in Fig. 9. ${ }^{C}$ The central component showed a clearly resolvable g-width which agreed well with that predicted by the q-dependence of $\omega_{\omega}^{2}$.

\section{DISCUSSION}

Fron the outset we have assumed that the scattering associated with the central mode is a part of the one-phonon response and is dynamical in nature, but with a narrow frequency response. However, in view of the. fact that we have not succeeded in demonstrating experimentally an energy width, it is important to

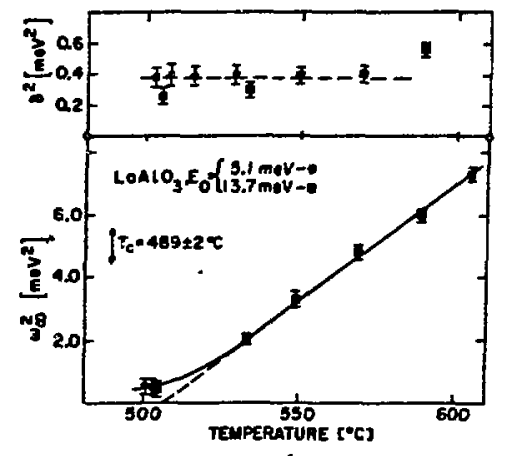

Neg. 非 2-346-73

Figure 9. $\delta^{2}$ and $\omega_{a}^{i}$ vs $T$ for $\mathrm{LaAlO}_{3}$. 
consider whether an alternative static mechanism could explain our observed central component. While it is true that the intensity of the central component near $T_{M}$ strongly suggests its dynamical orlgin, it is possible to Imagine a plausible and somewhat trivial nondynamical mechanism involving scattering from static strain flelds, which 18 at least in qualitative agreement with many of our observations. It is well known that point defects in a lattice will in general give rise to displacements of neighboring atoms from their equilibrium positions in the homogeneous impurity free crystal. These displacement flelds cause diffuse scattering of $x$-rays or neutrons sometimes known as Huang scattering. The magnitude of the displacement field about an $1 \mathrm{~m}-$ purity is calculated as a linear response to a force field $\vec{F}(\vec{r})$ which the impurity exerts on the undisplaced lattice. For our purposes it is conventent to Fourler transform the resulting displacements and express them in terms of a linear combination of phonon modes with wave vector $q$ and branch Index $\mathrm{j}$. The amplitude of this impurity induced set of static phonon-1ike displacements is easily shown in the harmonic approximation to be given by

$$
\left\langle Q_{q j}\right\rangle_{\operatorname{seatic}}=F_{q j} / \omega_{q j}^{2}
$$

where

$$
F_{q j}=-N^{-\frac{1}{2}} \sum_{l^{2}} M_{k}^{-\frac{1}{2}} \vec{F}_{l k} \cdot \vec{e}_{k}^{*}(q j) \exp (-1 \vec{q} \cdot \vec{z})
$$

fs the projection of $\overrightarrow{\mathbf{F}}(\vec{r}) \equiv \overrightarrow{\mathrm{F}}_{\ell k}$ upon the phonon eigenvector e $(q, j)$. The intensity of thisk static diffuse scattering can be calculated from the corresponding expression for the integrated phonon scattering under the same conditions by oimply replacing $\left\langle Q_{q j}^{2}\right\rangle_{\text {thermal }}$ by $\left|\left\langle Q_{q j}\right\rangle_{\text {static }}\right|^{2}$.

$$
I_{q j}(\vec{Q})_{\text {static impurity }} \propto \mid\left\langle Q_{q j^{\prime}} \text { static }\left.\right|^{2}=\frac{\left|F_{q j}\right|^{2}}{\omega_{q J}^{4}} .\right.
$$

In normal materials the major contributions come from long wavelength acoustic modes (because of the weighting by $\omega^{-4}$ ) and the effect of impurity concentrations of $210^{-\frac{2}{2}}$ can eastily be detected and studied against the thermal diffuse background by $x$-ray scattering. However, Eq. (7) also suggests that this same factor of $\omega^{-4}$ would greatly enhance the contribution of any

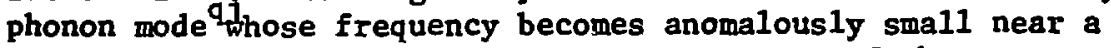
structural transformation. If impurities are of the proper symmetry to couple to the soft mode, there will be a central component, static in origin, whose intensity grows more rapidly $\left(\omega_{\text {soft }}^{-4}\right)$ than that of the collapsing phonon sidebands $\left(\omega_{80 f t}\right)$. 
In spite of thege obvious gimllaritles, we do not belleve that th1s impurity mèchanism provides a satisfactory explanation of our observations. Although we have not unt1l now made the distinction, it is clear that it is the low frequency stiffness $w^{2}$ which goes into Eq. (7) not $\omega_{\infty}^{2}$, If there is a difference between the two quantities. However, the static 1mpurity mechanism acting alone provides no frequency dependent terms to the phonon self-energy, so that $\omega_{0}^{2}=\omega_{\infty}^{2}$ and $I(Q)$ stats $\approx 1 / \omega_{\infty}^{4}$. For $\mathrm{Nb}_{3} \mathrm{Sn}$ our measurements closely follow $\left(1 / \omega^{2} \omega^{2}\right)$, and there is a substantial difference between the two predictions especially near $T_{Y}$. Simply put, $w^{2}$ (as obtained from the phonon sidebands) satutates near $T_{M}$ while the central intensity contiaues to increase. Also the observed agreenent with the discrepancy between the extrapolated long wavelength acoustic velocitles and our messurements and the magnitude of the central component would be entire1y fortuitous for this (or for that matter any other) static description of the central component.

We belleve that it $1 \mathrm{~s}$ unlikely that the impurity effect outlined above is the dominant one in the observations described here. On the other hand, ft is certainly a plausible mechanism for producing unusual line shape effects near phonon instaitilties and as such deserves further consideration. It is obvious from these comments that it is most important to try to characterize, directly if possible, the energy width of the central component. If the estimate of $\gamma * 10^{10} \mathrm{~Hz}(20.4$ meV) obtained 16 Indirectly from ESR date in $\mathrm{SrT}_{3} \mathrm{O}_{3}$ is correct, these widths are at the limit of conventional neutron resolution, and it is possible that light scattering is the more appropriate tool.

\section{REFERENCES}

1. T. Rigte, E. J. Sawuel Ben, and $K$. Otnes in "Structural Phase Trans1tions and Soft Modes," ed. E. J. Samuelsen, E. Andersen, and J. Feder (Universtetsforlaget, Cslo, Norway, 1971). See also, T. R1ste, E. J. Samuelsen, K. Otnes, and J. Feder, Solld State Commun. 9, 1455 (1971).

2. C. Shirane and J. D. Axe, Yhya. Rev. Letters 27, 1803(1971).

3. W. Rehwald, M. Rayl. R. W. Cohen, and G. D. Cody, Phys. Rev. (to be pubilshed). 'inls contalns references to earlier work.

4. R. Mallfort, B. W. Batterman, and J. J. Hanak, Phys. Lett. 24A, 315 (1967); L. J. Vieland, R. W. Cohen, and W. Rehwald, Phys. Rev. Letters 26, 373 (1971).

5. F. Schwabl, Phys. Rev. Letters 28, 500 (1972). 
6. T. Schnelder, to be published.

7. H. Unok1 and T. Sakuto, J. Phys. Soc. Japan 23, 546 (1969),

8. P. A. Fleury, J. F. Scott, and J. M. Worlock, Phys, Rev. Letters 21 , 15 (1968).

9. G. ShIrane and Y. Yarada, Phys. Rev. 177, 658 (1969).

10. S. M. Shapiro, J. D. kxe, G. Shirane, and T. R1ste, Phys. Rev. B 6, 4332 (1972).

11. K. Gest, J. D. Axe, and G. Sh1rane, Phys. Rev, D, 1933 (1972).

12. K. Otnes, T. RIste, G. Shirane, and J. Feder, Solid State Cormun. 9, 1103 (1971).

13. V. J. Hinkiewtez and G. Shirane, J. Phys. Soc. Japan 26, 674 (1969); G. Shirane, V. J. Minkiewicz, and A. Linz, Solld State Comoun. B, 1941 (1970).

14." W. Cochran and A. Z1a, Phys. Stat. Sol. 25, 273 (1968);

G. Shirane, and K. A. Wuller, Phys. Rey. J83, 820 (1969).

15. J. K. Kłems, G. Shirane, K. A. Muller, and H. J. Scheel, to be published.

16. Th. von Waldkirch, K. A. Muller, and W. Berlinger, Phys, Rev. 1. 1652 (1973). 